\title{
Development of the BeefSpecs fat calculator: a tool designed to assist decision making to increase on-farm and feedlot profitability
}

\author{
$\underline{\text { B.J. Walmsley }}^{\text {a,b }}$, V.H. Oddy ${ }^{\text {a,b }}$, M.J. McPhee ${ }^{\text {a,b }}$, D.G. Mayer ${ }^{\text {a,c }}$, and W.A. McKiernan ${ }^{\text {a,b }}$ \\ ${ }^{a}$ Cooperative Research Centre for Beef Genetic Technologies, ${ }^{b}$ Beef Industry Centre, New South Wales \\ Department of Primary Industries, Armidale, New South Wales, ${ }^{c}$ DEEDI, Dutton Park, Queensland. \\ Email: brad.walmsley@industry.nsw.gov.au
}

\begin{abstract}
The BeefSpecs fat calculator combines data from beef cattle growth-path studies with the extensive body of knowledge within animal growth and body composition models to predict body composition of cattle. It uses easy to obtain on-farm measurements to assist beef producers make management decisions to better achieve domestic and international market specifications. To facilitate uptake, it makes explicit use of practical end-user knowledge of cattle and production systems that can be translated for incorporation into the underpinning research models and output is returned in producer language.
\end{abstract}

The simple interface of BeefSpecs has been refined and developed to be used in a structured manner.

- Its primary use is as an educational tool to demonstrate the relationship(s) between management actions and the performance of groups of animals,

- The next level of use is to facilitate refined animal management on-farm by assisting drafting decisions to create sub-groups of animals according to expected performance, and in its final form,

- It will be used to optimise feeding and marketing decisions to increase profitability in both feedlots and pasture finishing systems.

BeefSpecs has evolved. Initially BeefSpecs (BeefSpecs1) was developed using an animal growth and body composition model called the Davis Growth Model (DGM) which requires an estimate of feed intake to operate. However accurate feed intake information is not a realistic input in commercial production systems. To overcome this, a wide variety of alternative scenarios were simulated using the DGM, and a multiple regression interpolation of the simulation results was used to predict P8 rump fat depth. The agreement between observed and predicted P8 fat depths using this approach in commercial cattle was relatively high. However, there were some circumstances where the multiple linear regression method produced poor agreement with observed P8 fat depths, especially when growth rates were below $0.5 \mathrm{~kg} / \mathrm{day}$.

To resolve this, phase two of BeefSpecs (BeefSpecs2) was implemented to directly use an alternative animal growth model (Meat Animal Research Centre model, MARC), that does not require feed intake as an input. A simplified version of the MARC model is used to predict the composition of empty body weight from animal growth rate $(\mathrm{kg} / \mathrm{day})$ given a description of animal type. Agreement between observed and predicted P8 fat depths in BeefSpecs2 was considerably better than for BeefSpecs1. Where BeefSpecs1 had problems predicting P8 fat depth at low growth rates, BeefSpecs2 has increased prediction robustness.

Refinements to the modeling systems underlying BeefSpecs have allowed development of increasingly sophisticated applications. A tool for on-farm drafting has been developed to assist producers explore the effects drafting animals into sub-groups to manage independently has on their capacity to meet market specifications. This tool is designed to work in association with a national mechanism that provides carcass feedback data following slaughter allowing the impacts of alternative management strategies on carcass traits to be to explored. Additional tools have been developed that target refinement of animal allocations to either pens or paddocks in feedlots and pasture finishing systems, with the purpose of reducing days to slaughter to increase overall production system profit. Work is also progressing to extend BeefSpec's capabilities to predicting retail meat yield and intramuscular fat content using on-farm measurements.

Keywords: Decision Support Systems (DSS), beef cattle, model development, market specifications, body composition 


\section{INTRODUCTION}

Beef producers continually make management decisions that impact on both the capacity of their cattle to meet market specifications and the profitability of their beef businesses. Recent analysis of 40,000 feedlot records has demonstrated that non-compliance of beef cattle to market specifications in the Australian beef industry is high (Slack-Smith et al., 2009). Analysis of 20,000 records for short fed cattle indicated that $28 \%$ missed carcass weight specifications costing $\$ 5.50 /$ head or $\$ 31,000$ and $16 \%$ missed P8 fat specifications costing $\$ 17.50 /$ head or $\$ 56,000$ (Slack-Smith et al., 2009). In addition, analysis of 20,000 records for long fed cattle reported that $29 \%$ missed carcass weight specifications costing $\$ 11 /$ head or $\$ 64,000$ and $70 \%$ missed the marbling specification of score 3 or better costing $\$ 105 /$ head or $\$ 1,470,000$.

The BeefSpecs fat calculator is a decision support tool that has been developed within the Cooperative Research Centre for Beef Genetic Technologies (Beef CRC) to assist producers address the issue of noncompliance (Walmsley et al., 2010). BeefSpecs combines the predictive powers of animal growth and compositional models (Davis Growth Model, DGM; Oltjen et al., 1986, Meat Animal Research Centre Model, MARC; Keele et al., 1992; Williams and Jenkins, 1998) with practical information relating to animal growth and fatness in response to changes in production environment. The tool assists beef producers to improve compliance with target market specifications when making management decisions that affect production by predicting end-point fatness. To achieve this, BeefSpecs is required to function across a wide range of production environments and have sufficiently simple user interaction to encourage on-farm use.

A major challenge in development of BeefSpecs has been to combine experimental data with the computational power of animal growth and body compositional models while using easy to obtain on-farm inputs. This has required an interpretive process to relate on-farm measures to the input parameters required within growth models and then to convert growth model outputs back into easily understood animal production language. Refinements to the underlying growth and body compositional models have allowed average daily gain (ADG; $\mathrm{kg} / \mathrm{day}$ ) to replace daily feed intake as the main driver of predicted fat deposition. Breed composition is input by an interactive visual comparison with live animals in the absence of accurate breed content. These refinements and the predictive capacity of the underlying models provide BeefSpecs with the ability to operate across a wide range of production environments while maintaining sufficiently simple user interaction to enable/encourage users to readily explore management options.

Solving such problems during the development of BeefSpecs has allowed new opportunities to surface. These include tools to assist in implementing higher level animal management systems e.g. on-farm assisted drafting and optimisation of cattle allocation during feedlot induction or on-farm mob formation. These downstream applications of the principles embodied in BeefSpecs are currently being tested in production systems. The prediction of intramuscular fat (IMF; marbling) and retail meat yield using on-farm measures are other developments that will add to the utility of BeefSpecs particularly as market specifications evolve.

This paper will describe the evolution of the BeefSpecs fat calculator from the conceptual stage to the current working version. Other tools that have been developed from the BeefSpecs framework will also be described.

\section{THE BEEFSPECS CALCULATOR}

\subsection{Original Development}

The dynamic steer growth model called the Davis Growth Model (DGM) (Oltjen et al., 1986) formed the base for the first phase of the BeefSpecs fat calculator (BeefSpecs1; Figure 1). The DGM describes the deposition of energy as lean and fat in the empty body in response to nutrient intake during an animal's growth (Oltjen et al., 1986). Subsequent additions include a description of partitioning of fat between fat depots in the body (e.g. subcutaneous, intermuscular, visceral and intramuscular) (McPhee et al., 2007; Sainz and Hasting, 2000). To predict industry relevant fat deposition from the DGM the quantity of total body fat is used to estimate subcutaneous fat, which in turn is converted to millimetres of fat thickness at the 12/13th rib site (McPhee et al., 2008) and to millimetres of fat thickness at the P8 site after taking sex, breed type and live weight into consideration (Walmsley et al., 2010). Inputs used to initialise the DGM growth model include frame score (height at the hips relative to age - as a measure of animal maturity), initial P8 fatness and initial live weight (an assessment of current phenotypic status). Implant (hormonal growth promotant, HGP) status, days on feed, sex, and breed type are used to drive predictions of fat deposition in conjunction with a description of the feed quality and quantity eaten. 
The need for an estimate of feed intake to run the DGM represented an obstacle to its direct application, as farmers usually don't have this information. To work around this, a series of simulations were run with the DGM to produce a matrix of inputs and outputs that covered a range of initial weights, frame scores, initial fat depths, implant status, days on feed, along with metabolisable energy content of the feed and intake. This allowed a multiple linear regression to be developed from this matrix of inputs and outputs that operates directly behind the BeefSpecs calculator. The prediction of $\mathrm{P} 8$ fat depth was consequently driven primarily by $\mathrm{ADG}$ in response to other inputs entered into the calculator (McKiernan et al., 2008). The metabolisable energy content of the feed, and animal maintenance energy requirement was replaced in the multiple regression framework with a description of the production system in terms of quality of feed, distance to water and surrounding terrain on the scale 1 to 4 (e.g. 1: feedlot/strip grazing vs. 4: large paddocks, native pasture, hilly terrain). Inputs relating to animal type that are located on the 'Animals tab' are demonstrated in Figure 1 along with the results section. The inputs

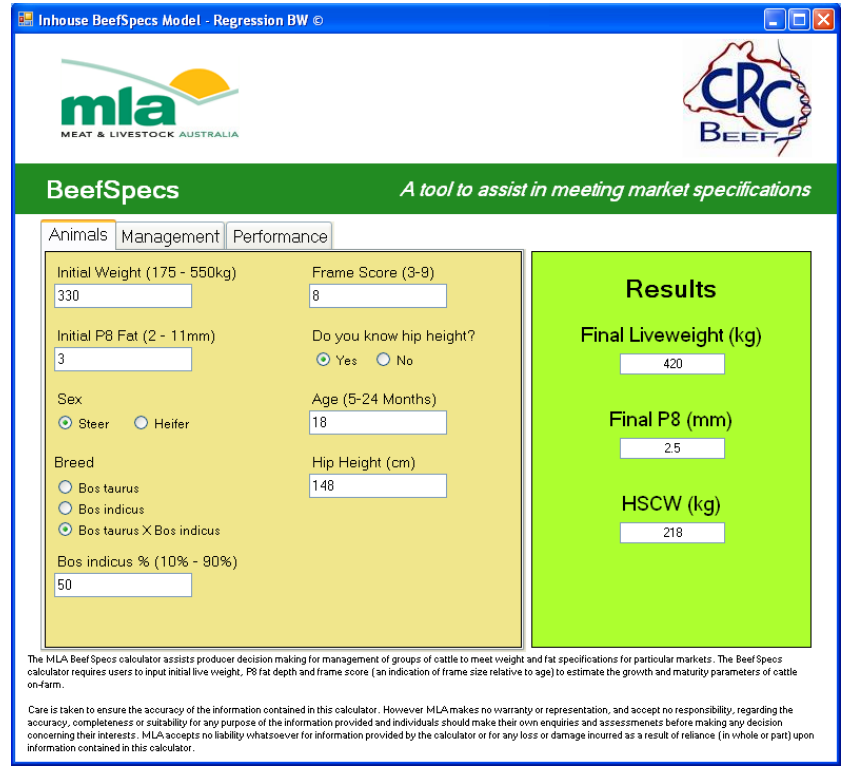

Figure 1. The BeefSpecs user interface developed during phase one that shows the inputs for frame score, sex, breed, initial weight and P8 fat as well as the predicted outputs (under 'results' heading). entered into and the outputs obtained from the BeefSpecs fat calculator are group averages as opposed to individual animal measures, because of the nature of the data sets used to derive the model parameters.

\subsection{Refinement to Develop Current BeefSpecs}

The second phase of BeefSpecs development (BeefSpecs2; Figure 2) has been conducted in response to shortcomings identified in predictions using the multiple linear regressions derived from DGM simulations. These are discussed below in section 2.3. BeefSpecs2 uses a growth model directly behind the calculator. To overcome the problem of users not knowing feed intake, the Meat Animal Research Centre (MARC) model originally developed by Keele et al. (1992) and subsequently refined by Williams and Jenkins (1998) was modified to fit within the BeefSpecs interface. The MARC model predicts the composition of empty body weight from animal growth rate (kg/day) given a description of animal type (frame score, sex and breed type), period of feeding (days) and initial conditions (initial weight and P8 fat). The MARC model is based on the observation that an animal of a given type will, on average, have a defined body composition (fat and lean) when treated in a specified manner while growing at a particular rate. The modified MARC model predicts rib fat depth $(\mathrm{mm})$ from simulated total body fat. Rib fat depth is used to predict P8 fat depth (mm) taking account of sex, breed type and weight of the animal (Walmsley et al., 2010).

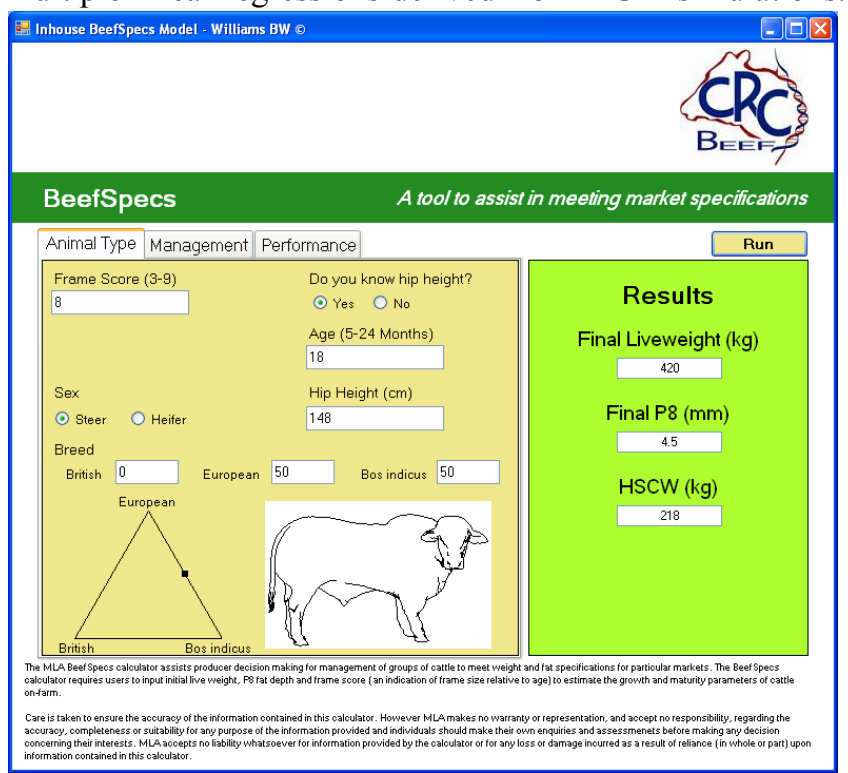

Figure 2. The BeefSpecs user interface developed during phase two illustrates how frame score, sex and the visual method used to describe breed are included on the 'Animal Type' tab. 
The effects that prevailing production conditions (growth hormone treatment, and feed type [grass vs. grain]) have on body composition have been incorporated into the modified MARC model. A lagged response in growth and body composition to HGPs has also been implemented in the MARC model to reflect observed production responses (Oltjen et al., 1986). Grass fed animals are leaner than grain fed animals growing at the same rate (Tudor, 1992) and this has also been implemented in the revised MARC model.

The simple interface and user friendly attributes of BeefSpecs1 have been maintained in BeefSpecs2. Differences include: specification of production system has been removed, feed quality is accounted for by the growth rate and feed type (grass vs. grain) inputs, and breed type description has been expanded to include Bos indicus, British and European breed types and their crosses. Breed type is accommodated by a dynamic visual comparison with the live animal on the user input interface (Figure 2). Re-arrangement of the BeefSpecs interface has occurred to better differentiate inputs under the categories of 'Animal Type', 'Management' and 'Performance' to reflect the impacts they have on animal growth and composition (Figure 2). 'Animal Type' is a description that sets the production potential of the animal given ideal conditions. 'Management' refers to how this potential can be manipulated by altering aspects of the production system. 'Performance' is a description of where the animal starts (e.g. initial weight and fat) and the performance it exhibits (e.g. growth rate, days on feed).

\subsection{How do the different versions of BeefSpecs compare?}

The accuracy of P8 fat predictions made by different versions of the BeefSpecs fat calculator has been evaluated using 31 datasets that contain 3505 animals. A number of these datasets $(n=10)$, including some with multiple observations, are used here to demonstrate the improvement in accuracy achieved in BeefSpecs2 compared to BeefSpecs1. The data have been collected from a variety of production scenarios. These range from animals grown in feedlot conditions under experimental protocols to those grown in feedlots under commercial conditions to animals grown and fattened on pasture under standard industry practices.

BeefSpecs1, based on the multiple regression of the DGM, has been demonstrated to generally Table 1. Comparison of predicted and observed P8 fat $(\mathrm{mm})$ presented with mean bias (predicted - observed) for the two phases of BeefSpecs.

\begin{tabular}{lccccc}
\hline & & \multicolumn{2}{c}{ BeefSpecs 1 } & \multicolumn{2}{c}{ BeefSpecs 2 } \\
\cline { 3 - 6 } Dataset & $\begin{array}{c}\text { Observed, } \\
\mathrm{mm}\end{array}$ & $\begin{array}{c}\text { Predicted, } \\
\mathrm{mm}\end{array}$ & $\begin{array}{c}\text { Mean Bias, } \\
\mathrm{mm}\end{array}$ & $\begin{array}{c}\text { Predicted, } \\
\mathrm{mm}\end{array}$ & $\begin{array}{c}\text { Mean Bias, } \\
\mathrm{mm}\end{array}$ \\
\hline Albury & 6.64 & 10.66 & -4.02 & 6.39 & 0.25 \\
Ben Lomond & 7.44 & 6.29 & 1.15 & 5.93 & 1.51 \\
Glen Innes & 9.68 & 11.84 & -2.16 & 9.61 & 0.06 \\
Holbrook & 10.80 & 6.79 & 3.97 & 10.49 & 0.26 \\
Morven & 10.33 & 9.53 & 0.80 & 9.97 & 0.36 \\
Wagga Wagga & 10.97 & 17.64 & -6.67 & 12.43 & -1.45 \\
Willowtree & 8.84 & 7.63 & 1.21 & 9.39 & -0.55 \\
Werribee & & & & & \\
- Jan to Mar & 4.85 & 4.17 & 0.68 & 4.79 & 0.07 \\
- Mar to May & 5.57 & 2.35 & 3.23 & 5.69 & -0.11 \\
FLOT210 & & & & & \\
- 1 $^{\text {st }}$ Period & 12.63 & 15.81 & -3.18 & 13.24 & -0.62 \\
- 2 2 $^{\text {nd }}$ Period & 23.19 & 25.77 & -2.58 & 21.62 & 1.57 \\
Feedlot & & & & & \\
- April & 17.99 & 19.05 & -1.06 & 18.58 & -0.59 \\
- October & 19.54 & 20.45 & -0.92 & 21.76 & -2.22 \\
- December & 18.62 & 19.96 & -1.35 & 20.37 & -1.75 \\
\hline
\end{tabular}

display close agreement

between predicted and measured P8 fat depths (Table 1). However, there are a few notable exceptions. When animals are grown at low growth rates on pasture (below $\sim 0.5 \mathrm{~kg} /$ day) BeefSpecs1 had a tendency to make large under-predictions of P8 fat depth (e.g. Werribee - March to May) whereas when growth rates were higher (above $\sim 0.5 \mathrm{~kg} /$ day) predictions were more accurate (e.g. Werribee - January to March). When animals were grown for extended periods of time (> 200 days) BeefSpecs1 tended to make large overpredictions of P8 fat (e.g. Wagga Wagga).

Predictions of fatness for the same animals using BeefSpecs2, which is based on the MARC model, are also shown in Table 2. The predictions of P8 fat depth made by BeefSpecs2 showed close agreement with observed P8 fat depths and were much better overall than predictions by BeefSpecs $1-11$ of 14 cases had lower mean bias with the remaining 3 having little practical difference (Table 2). The two problems highlighted above for cases of low growth rates (e.g. Werribee - March to May) or growth over extended periods of time (e.g. Wagga Wagga) were eliminated or greatly reduced by the use of BeefSpecs2. 


\section{FUTURE DIRECTIONS OF BEEFSPECS}

The discussion above illustrates that during development and implementation, practical computer models evolve to meet the needs of the end-users and to correct discrepancies invoked due to imperfect implementation. However, there are further opportunities not yet fully exploited that arise from the successful uptake of a tool whose structure embodies knowledge of how animals grow. Although invisible to the enduser, the research model underpinning BeefSpecs embodies biological and physical rules about animal growth and development that easily permit expansion to address new opportunities. For example, BeefSpecs is being expanded to include predictions of boning room meat yield and intramuscular fat content, both of which directly impact on specification and price received for product. Implementation will be as an extension of the current product, because these new outputs require the same on-farm inputs for estimation. By building BeefSpecs over peer reviewed scientific / biological models such extensions are relatively easy to implement, provided that data to link the practical inputs and outputs is available or at least able to be collected.

This process is expected to continue in the future with development of other management tools based on the BeefSpecs framework. The on-farm drafting and feedlot/on-farm optimisation tools are described below.

\subsection{On-Farm Drafting}

The aim of this extension of BeefSpecs is to facilitate producer exploration of management systems to better achieve specifications by dividing animals into groups that may require different management strategies to meet targets. To implement this, a simple tool has been developed to the testing stage. It uses the BeefSpecs framework of generating average predictions of future P8 fat depth based on individual weight, current P8 fat depths, expected growth rates and production conditions for groups of animals. It then sorts animals into

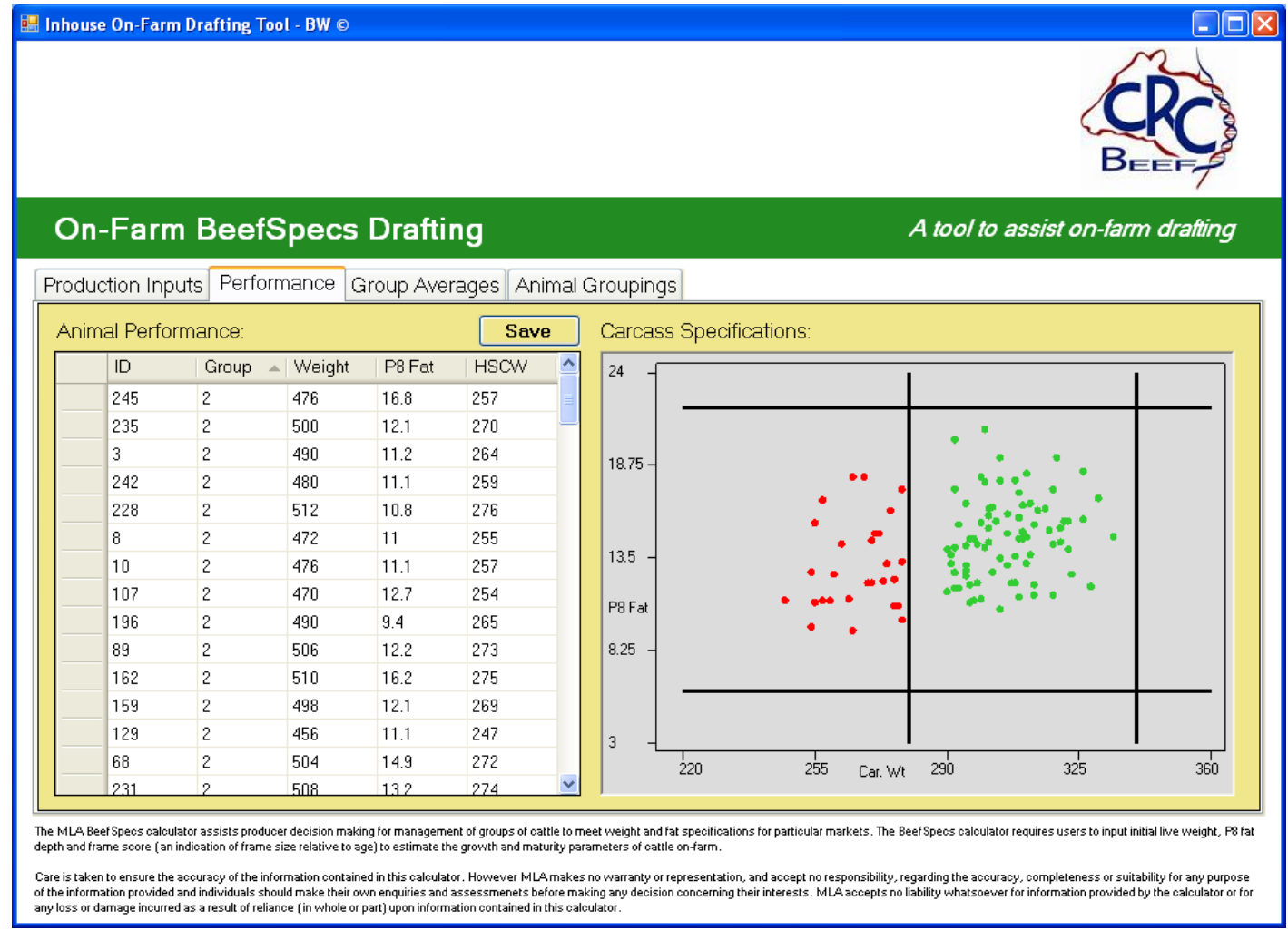

Figure 3. On-farm drafting interface demonstrating P8 fat and hot standard carcass weight predictions for individuals (left) and how these compare to market specifications (right). Data points are coloured according to their compliance with market specifications: green $=$ compliant and red $=$ non-compliant.

expected final outcomes and matches these against market specifications (Figure 3). This enables producers to explore the predicted impacts that management changes will have on the performance of sub-groups independent of the whole group average. This development has been achieved without alteration to the underlying prediction equations of the MARC model or changes to the input information required from the user to run the model. The only additional information required is the market specifications being targeted i.e. P8 fat depth and carcass weight. The drafting tool has strong synergies with an emerging national standardised carcass feedback mechanism associated with the National Livestock Identification System 
(NLIS). This mechanism provides carcass feedback data following slaughter to bench mark compliance rates. Interaction with this mechanism provides the opportunity to extend the use of the drafting tool to explore the impacts alternative management options could have on outcomes in the carcass.

\subsection{Feedlot/On-Farm Optimisation}

Mayer et al. (2007) used the predicted outputs of BeefSpecs1 in conjunction with an optimisation engine to allocate animals to feedlot pens based on compliance with market specifications. The optimised allocation was compared to a base scenario, a scenario where animals were penned by breed and a scenario where animals were penned by initial live weight, using a single cohort of 306 steers from the Beef CRC 'Regional Combinations' experiment (McKiernan et al., 2005). The optimisation indicated that penning animals based on how well and when they met market specifications increased feedlot gross margin by $\$ 2,200$ or $3.0 \%$ compared to the base scenario. This increase in gross margin was greater than that seen when grouping animals by breed or initial live weight. This successful illustration that BeefSpecs can improve profit led to the development of

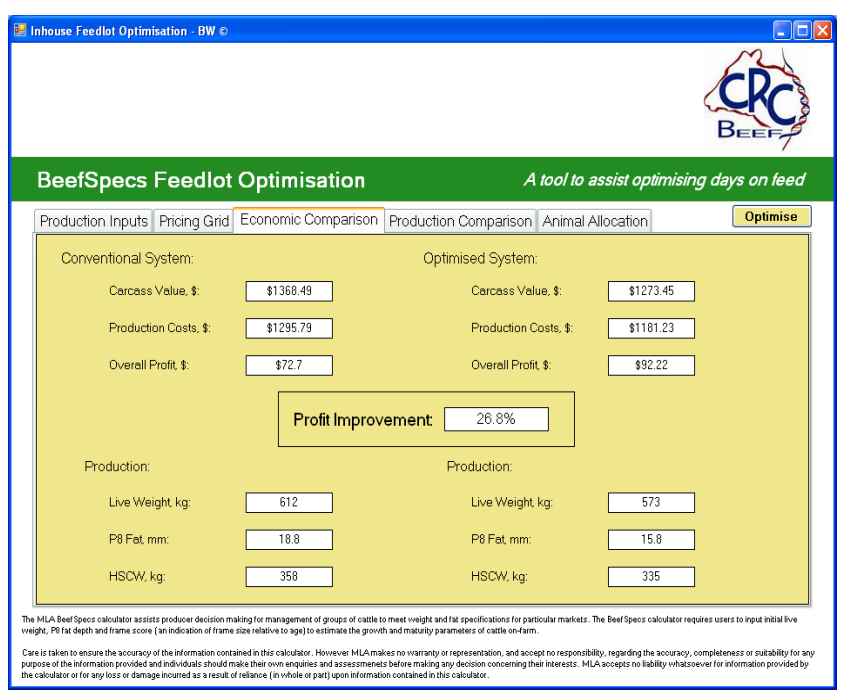

Figure 4. Economic comparison of conventional vs. optimised pen allocation from the tool designed to assist feedlots optimise pen allocation to reduce days on feed. decision-rules that underpin the BeefSpecs2 framework in the optimisation tool. This tool allocates animals to different pens based on predictions of individual P8 fat levels following nominated growth and management conditions. The aim is to refine pen allocations to allow optimal time on feed required to reach defined market specifications and thereby minimise production costs and maximise profit (Figure 4). While the increase in profit predicted by the initial study (Mayer et al., 2007) was rather modest, much greater potential has been shown in subsequent developments of the tool. The principles contained in the feedlot optimisation tool can also be applied in grazing situations to allocate animals to groups which have a similar propensity to meet market specifications. On-farm application of the optimisation tool has required some refinement to take into account the number of available paddocks and prevent long-term maintenance of animals given the comparatively low cost of feed derived from pasture.

\subsection{Implementation}

The principles behind the BeefSpecs fat calculator are sufficiently general to permit implementation at several levels. The base BeefSpecs calculator has the capacity to facilitate educational processes by demonstrating the broad range of effects that management decisions have on average group performance. When used appropriately in conjunction with other skills, such as live animal assessment, the BeefSpecs calculator can also provide guidance for decision-making processes. To facilitate uptake, and obtain the benefits from, BeefSpecs in the wider beef industry a series of pilot studies are being conducted in New South Wales. These studies aim to gauge and develop the knowledge and skill levels of producers using BeefSpecs to aid decision-making. More direct applications of BeefSpecs principles are aimed at implementation of the on-farm drafting and optimisation tools. Although these tools are at preliminary stages of development, they clearly demonstrate the potential of using information from individual animals in a dynamic nature to make decisions concerning sub-groups of animals to benefit the whole production system. Both tools have the capacity to link to a laser capture system that is under development to automatically record hip heights and thus frame scores (Wilkins et al., 2008; 2009). Such an initiative will increase the efficiency with which animals can be processed and decisions made regarding management, both on-farm and at feedlot induction.

\section{CONCLUSIONS}

The BeefSpecs fat calculator was designed to combine the knowledge obtained from growth path experiments with that contained in animal growth and body composition models, to assist beef producers 
make management decisions that enable their cattle to better meet market specifications. BeefSpecs has undergone two stages of development that have seen improvements in the prediction of P8 fat depths under different production circumstances. Other tools have been developed, using the same underlying principles, to extend the capability of this technology. These tools use predictions of individual animal performance to form sub-groups management purposes, where appropriate, rather than using average group inputs and outputs. These types of developments are expected to continue to occur into the future as BeefSpecs and other such tools are used under commercial production conditions to allow refinements to maintain/optimise productivity and economic viability of the beef industry.

\section{ACKNOWLEDGMENTS}

The authors gratefully acknowledge the in-kind and funding support from NSW Department of Primary Industries and the Cooperative Research Centre for Beef Genetic Technologies. The assistance of NSW Department of Primary Industries Beef extension officers and commercial feedlot operations in the collection of data has been invaluable in testing of the BeefSpecs calculator and development of future applications. Two anonymous reviewers are also thanked for their comments and suggestions.

\section{REFERENCES}

Keele JW, Williams CB, and Bennett GL. 1992. A computer model to predict the effects of level of nutrition on composition of empty body gain in beef cattle: I. Theory and development. Journal of Animal Science 70: 841-857.

Mayer DG, McPhee MJ, Oddy VH, Kinghorn BP, Oltjen JW, McKiernan WA, and Wilkins JF. 2007. Evolutionary computation targeting market fat specifications in beef steers. In: 'MODSIM 2007 International Congress on Modelling and Simulation.' (L. Oxley and D. Kulasiri eds.). Modelling and Simulation Society of Australia and New Zealand. University of Canterbury, Christchurch, New Zealand. p 81-87.

McKiernan WA, Wilkins JF, Barwick SA, Tudor GD, McIntyre BL, Graham JF, Deland MPB, and Davies L. 2005. CRC 'Regional Combinations' project - effects of genetics and growth paths on beef production and meat quality: Experimental design, methods and measurements. Australian Journal of Experimental Agriculture 45: 959-969.

McPhee MJ, Oltjen JW, Fadel JG, and Sainz RD. 2007. Simulation model of fat deposition and distribution in beef steers: 3. Model description and development. Journal of Animal Science. (Suppl1.), 85:609.

McPhee MJ, Oltjen JW, Fadel JG, Perry D, and Sainz RD. 2008. Development and evaluation of empirical equations to interconvert between twelfth-rib fat and kidney, pelvic, and heart fat respective fat weights and to predict initial conditions of fat deposition models for beef cattle. Journal of Animal Science 86: 1984-1995.

Oltjen JW, Bywater AC, Baldwin RL, and Garrett WN. 1986. Development of a dynamic model of beef cattle growth and composition. Journal of Animal Science 62: 86-97.

Sainz RD, and Hasting E. 2000. Simulation of the development of adipose tissue in beef cattle. In: 'Modelling nutrient utilisation in farm animals.' (JP McNamarra, J France and DE Beever eds.) CAB International, Wallingford, U.K. p 175-182.

Slack-Smith AR, Griffith GR, and Thompson JM. 2009. The cost of non-compliance to beef market specifications. Australasian Agribusiness Review 17: 178-190.

Tudor GD 1992. Effect of diet on fat deposition in cattle. In: 'Proceedings of the Australian Society of Animal Production.’ No. 19. La Trobe University, Melbourne. p 89.

Walmsley BJ, Oddy VH, McPhee MJ, Mayer DG, and McKiernan WA. 2010. BeefSpecs a tool for the future: On-farm drafting and optimising feedlot profitability. Australian Farm Business Management Journal 7: 29-36.

Walmsley BJ, Wolcott ML, and McPhee MJ. 2010. Modelling the relationship between scanned rump and 12th-rib fat in young temperate and tropical bovines: Model development and evaluation. Journal of Animal Science 88: 1848-1859.

Wilkins JF, McKiernan WA, Littler B, and Driver T. 2009. Laser technology to enhance live cattle assessment. In: 'Industry and Investment NSW Beef and Sheep Conference', Orange Agricultural Institute, Orange, NSW. p 3.

Wilkins JF, McKiernan WA, Littler B, Thompson JM, and Driver T. 2008. Automatic capture of live conformation in cattle using laser technology. In: 'NSW DPI Sheep and Beef Conference', Orange Agricultural Institute, Orange, NSW. p 250-252.

Williams CB, and Jenkins TG. 1998. A computer model to predict composition of empty body weight changes in cattle at all stages of maturity. Journal of Animal Science 76: 980-987. 